\title{
A new approach to mapping the atmospheric effect for GPS observations
}

\author{
Ioannis M. Ifadis \\ Aristotle University of Thessaloniki, School of Civil Engineering, Division of Geotechnical Engineering, Section of Geodesy
}

(Received December 25, 1999; Revised July 17, 2000; Accepted July 31, 2000)

\begin{abstract}
In space to earth geodetic techniques the atmosphere affects the radio wave propagation. In order to estimate the total non-zenith path delay several mapping functions have been proposed to scale the zenith delay to other elevations. For all of them the basic idea is that the ultimate direction of the ray path is exactly that of the vacuum elevation of the radio source, and this is used to estimate the delay. This is true when we consider VLBI observations from a distant radio source but not the case for satellites or other near-earth radio sources. In this case the radio source distance is no longer infinite, and we have to think of a slightly different radio ray direction. This may insert a reasonable error in delay estimation. Thus, for satellite observations, the source elevation angle must be corrected for the ray bending effect before we use it with a mapping function to scale the delay. This means that either we have to estimate this correction of the angle or we have to develop a model that includes the correction. In this report we evaluate the magnitude of the correction and discuss the way we can approach and map it. Another purpose of this report was to study different mapping functions used to predict the hydrostatic delay at low elevation angles. Also a study of models with constant terms shows that such a model optimized for a site or region can give satisfactory results.
\end{abstract}

\section{Introduction}

The atmospheric delay for directions not too far from zenith may be approximated by the zenith delay multiplied by the cosecant of the elevation angle.

$$
\Delta L(\epsilon)=\Delta L\left(90^{\circ}\right) / \sin (\epsilon)
$$

This relation will obviously result in an increasing error with decreasing elevation angle because even if the atmosphere is thin, the earth cannot be assumed to be planar. In general we can write

$$
\Delta L(\epsilon)=\Delta L\left(90^{\circ}\right) m(\epsilon)
$$

For the past twenty years the most popular form of the mapping function, $m(\epsilon)$, was that proposed by Marini that has the form

$$
m(\epsilon)=\frac{1}{\sin (\epsilon)+\frac{a}{\langle\sin (\epsilon) \mid \tan (\epsilon)\rangle+\frac{b}{\sin (\epsilon)+\frac{c}{\sin (\epsilon)+\cdots}}}}
$$

where $\epsilon$ is the vacuum elevation of the radio source (deep space or satellite). But what is the correct elevation? Currently we use the vacuum elevation of the radio source at the moment of observation. But if we recall from the ray tracing techniques (used to calculate the atmospheric delays and the radio bending) what we really get is the elevation of the final point of integration. Thus, to our point of view there it is one more source of error. In this report we test

Copy right $(\mathrm{C})$ The Society of Geomagnetism and Earth, Planetary and Space Sciences (SGEPSS); The Seismological Society of Japan; The Volcanological Society of Japan; The Geodetic Society of Japan; The Japanese Society for Planetary Sciences. known mapping functions for this possible source of error. In Table 1 there are some results showing the effect of this error for GPS satellites and low orbit radio sources, where

- $\epsilon$ : apparent elevation angle at the antenna point (degrees)

- wet: wet delay (m)

- hyd : hydrostatic delay (m)

- total: total atmospheric delay (m)

- $p_{1}$ : error in the delay due to pointing angle mismodeling for GPS

- $p_{2}$ : error in the delay due to pointing angle mismodeling for a signal coming from a low orbit radio source (at the $100 \mathrm{~km}$ level: theoretical approximation).

\section{Hydrostatic Delay Mapping Functions}

Several investigators have proposed functions to scale the zenith hydrostatic delay to lower elevation angle. Some of them, which will be used later for the purposes of this report, are given briefly in the following.

Marini (1972)

$$
\Delta L=\frac{1}{f(\phi, h)} \frac{A+B}{\sin (\epsilon)+\frac{B}{(A+B)(\sin (\epsilon)+0.015)}} .
$$

Where the $A$ and $B$ are tabulated values.

Chao (1973) replaced the second $\sin (\epsilon)$ with $\tan (\epsilon)$, thus ensuring that $\Delta L(90)=0$ and used constant values for the function parameters. This formula was proposed for eleva- 
Table 1. Results for the delay mismodelling using ray tracing analysis for a typical temperate station $(\mathrm{m})$

\begin{tabular}{cccccc}
\hline$\epsilon$ & wet & hyd & total & $p_{1}$ & $p_{2}$ \\
\hline 10.0 & 0.4478 & 13.0299 & 13.4777 & 0.000 & 0.017 \\
5.00 & 0.8755 & 24.2713 & 25.1468 & 0.002 & 0.129 \\
4.00 & 1.0797 & 29.1146 & 30.1943 & 0.004 & 0.221 \\
3.00 & 1.4021 & 36.1186 & 37.5207 & 0.009 & 0.416 \\
2.00 & 1.9741 & 46.9215 & 48.8956 & 0.023 & 0.881 \\
1.00 & 3.1791 & 65.0979 & 68.2770 & 0.071 & 2.177 \\
0.50 & 4.3445 & 79.2981 & 83.6426 & 0.136 & 3.685 \\
0.00 & 6.2661 & 99.3908 & 105.6569 & 0.273 & 6.532 \\
\hline
\end{tabular}

tions down to $10^{\circ}$.

$$
\Delta L(\epsilon)=\frac{\Delta L\left(90^{\circ}\right)}{\sin (\epsilon)+\frac{0.00143}{\tan (\epsilon)+0.0445}}
$$

Davis et al. (1985) added one more term in the Chao function keeping $\tan (\epsilon)$ in the second. He introduced the use of the temperature lapse rate and the tropopause height $\left(a_{t}\right.$ and $\left.h_{t}\right)$ in the parameterization of the mapping functions.

$$
m(\epsilon)=\frac{1}{\sin (\epsilon)+\frac{a}{\tan (\epsilon)+\frac{b}{\sin (\epsilon)+c}}}
$$

where

$$
\begin{aligned}
& a, b=f\left(p, t, e, a_{t}, h_{t}\right) \\
& c=\text { constant }
\end{aligned}
$$

Ifadis (Ifadis, 1986, 1987) used a third order fraction form for the development of his models in the form of global and climate solutions. The proposed function is:

$$
m(\epsilon)=\frac{1}{\sin (\epsilon)+\frac{a}{\sin (\epsilon)+\frac{b}{\sin (\epsilon)+0.078}}}
$$

where $m(90)=1$ by definition and

$$
\begin{aligned}
& a=k 1+k 2\left(P_{0}-1000\right)+k 3\left(T_{0}-15\right)+k 4 \sqrt{ } p w \\
& b=k 1+k 2\left(P_{0}-1000\right)+k 3\left(T_{0}-15\right)+k 4 \sqrt{ } p w
\end{aligned}
$$

Herring (Herring, 1992-MTT model) changed somehow the basic form of the Marini fraction form introducing also a fraction form for the numerator of the mapping function. He proposed the function

$$
m(\epsilon)=\frac{1+\frac{a}{1+\frac{b}{1+c}}}{\sin (\epsilon)+\frac{a}{\sin (\epsilon)+\frac{b}{\sin (\epsilon)+c}}}
$$

where

$$
\begin{gathered}
a=\left[1.2320+0.0130 \cos \varphi-0.0209 H_{\sigma}\right. \\
\left.+0.00215\left(T_{s}-10\right)\right] 10^{-3}
\end{gathered}
$$

$$
\begin{gathered}
b=\left[3.1612-0.1600 \cos \varphi-0.0331 H_{s}\right. \\
\left.+0.00206\left(T_{s}-10\right)\right] 10^{-3} \\
c=\left[71.244-4.293 \cos \varphi-0.149 H_{s}\right. \\
\left.-0.0021\left(T_{s}-10\right)\right] 10^{-3}
\end{gathered}
$$

Niell, (Niell, 1996-NMF model) kept the form of the Herring formula but added a height correction term. He assumed that the elevation dependence is a function of only geographical parameters (latitude and height above sea level) and day of the year and proposed the function

$$
m(\epsilon)=\frac{1+\frac{a}{1+\frac{b}{1+c}}}{\sin (\epsilon)+\frac{a}{\sin (\epsilon)+\frac{b}{\sin (\epsilon)+c}}}+H_{s} 10^{-3} M_{h}
$$

where

$$
\begin{aligned}
& M_{h}=\frac{1}{\sin (\epsilon)}-\frac{1+\frac{a_{h t}}{1+\frac{b_{h t}}{1+c_{h t}}}}{\sin (\epsilon)+\frac{a_{h t}}{\sin (\epsilon)+\frac{b_{h t}}{\sin (\epsilon)+c_{h t}}}} \\
& \begin{array}{l}
a, b, c=f(\varphi, h, \text { day }) \quad \text { and } \\
a_{h t}, b_{h t}, c_{h t}=\text { constants }
\end{array}
\end{aligned}
$$

\section{Models' Characteristics and Discussion}

All of the above models have in common: the basic form of the continuous fraction which is used for the geometrical approximation of the elevation dependence of the delay. However they use quite different approach to modeling the fraction form parameters.

The Chao model is the simplest of all and uses only constant terms with no weather or site dependence. The Marini, and Herring (MTT) models use site latitude as the basic parameter but include also atmospheric parameters. The Niell (NMF) model is based only on the site characteristics longitude and height and has no atmospheric dependence. The Ifadis models are based only on atmospheric parameters (pressure, temperature, and water vapor pressure).

These approaches lead to different results concerning the behavior and accuracy of each one of the models. There are some points we have to discuss here. With reference to Table 4 it is interesting to see that the Chao model even the simplest of all performs well to low elevation angles. Then perhaps another model with constant terms and with better estimation will do even better. Such a model could also be a "Niell" model which will use only geographical parameters as site latitude and site altitude. But what has been shown (Ifadis and Savvaidis, 1999) is that for low elevation angles could also be a longitude dependence. Also we have to point out that geographical parameters can represent (perhaps) in the mean the pressure and temperature variations, but then what happens with the wet part of delay. The use of atmospheric parameters makes the model more "difficult in use" while the pressure component is proved to be "weak". The use of temperature lapse rate and the tropopause height needs exact information about the atmospheric profile, or the use of 
Table 2. $k_{I}$ coefficients for the Ifadis-86 hydrostatic delay mapping function.

\begin{tabular}{lccccc}
\hline \multirow{2}{*}{ global } & & $k 1$ & $k 2$ & $k 3$ & $k 4$ \\
& $\mathrm{a}$ & $0.12366410^{-2}$ & $0.13156610^{-6}$ & $0.13781710^{-5}$ & $0.80574910^{-5}$ \\
arctic & $\mathrm{b}$ & $0.33330510^{-2}$ & $0.19455610^{-6}$ & $0.10396510^{-5}$ & $0.17465810^{-4}$ \\
& $\mathrm{a}$ & $0.12185910^{-2}$ & $0.26350510^{-6}$ & $0.89180110^{-6}$ & $0.12812110^{-4}$ \\
\multirow{3}{*}{ temperate } & $\mathrm{b}$ & $0.33205810^{-2}$ & $0.54255010^{-6}$ & $0.56765910^{-6}$ & $0.23687610^{-4}$ \\
& $\mathrm{a}$ & $0.12334610^{-2}$ & $0.26737610^{-6}$ & $0.14277510^{-5}$ & $0.88683710^{-5}$ \\
tropic & $\mathrm{b}$ & $0.33345610^{-2}$ & $0.38004510^{-6}$ & $0.12843610^{-5}$ & $0.16464010^{-4}$ \\
& $\mathrm{a}$ & $0.12587610^{-2}$ & $0.15954810^{-6}$ & $0.83077910^{-6}$ & $0.46069910^{-5}$ \\
steppe & $\mathrm{b}$ & $0.33554310^{-2}$ & $0.20492610^{-6}$ & $0.67259410^{-6}$ & $0.14014910^{-4}$ \\
& $\mathrm{a}$ & $0.12309210^{-2}$ & $0.12240810^{-6}$ & $0.13210910^{-5}$ & $0.89568010^{-5}$ \\
desert & $\mathrm{b}$ & $0.33470310^{-2}$ & $0.25852610^{-6}$ & $0.11013510^{-5}$ & $0.15152510^{-4}$ \\
& $\mathrm{a}$ & $0.12688710^{-2}$ & $0.30698010^{-6}$ & $0.11852910^{-5}$ & $0.60517910^{-5}$ \\
mountain & $\mathrm{b}$ & $0.33715210^{-2}$ & $0.36967310^{-6}$ & $0.81823810^{-6}$ & $0.12671210^{-4}$ \\
& $\mathrm{a}$ & $0.12474510^{-2}$ & $0.82316410^{-6}$ & $0.12918310^{-5}$ & $0.11636710^{-4}$ \\
& $\mathrm{~b}$ & $0.33584710^{-2}$ & $0.12156410^{-5}$ & $0.13284310^{-5}$ & $0.19881710^{-4}$ \\
\hline
\end{tabular}

\section{Map with approximate positions of stations used in this report Most of the stations are from the northern hemisphere}

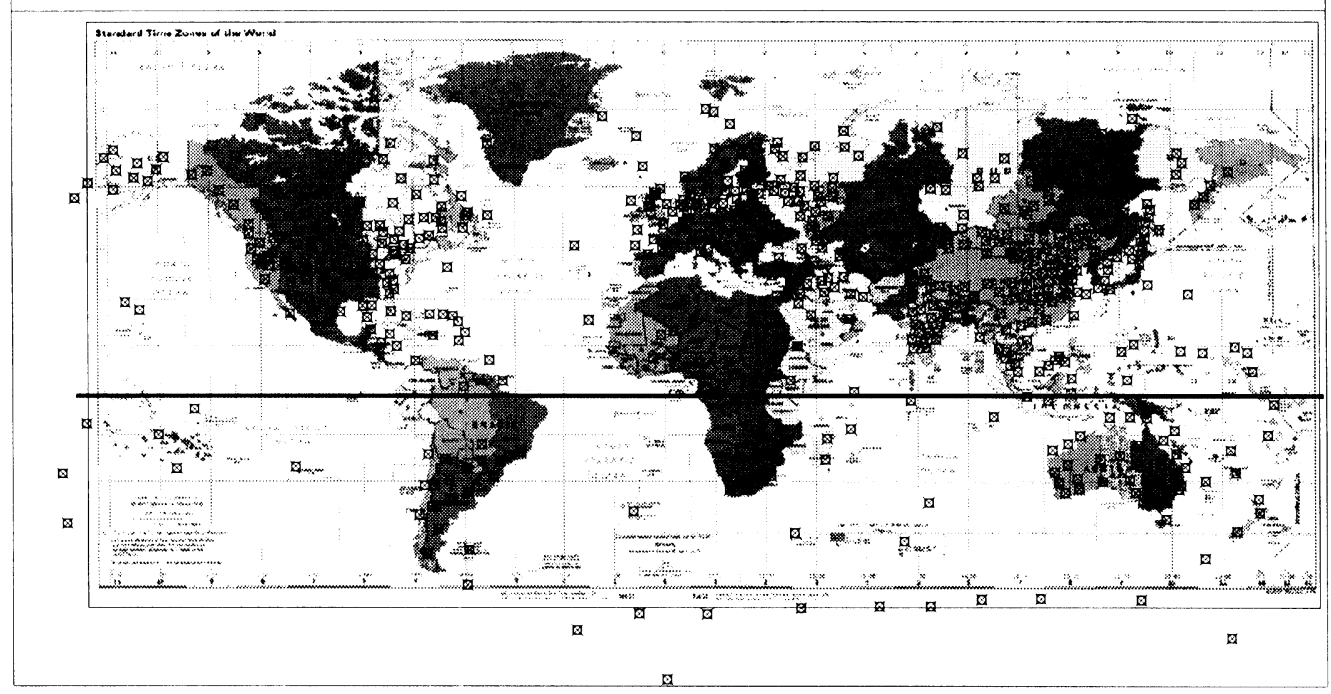

Fig. 1. Approximate global distribution of the stations used in this report.

another function to predict them, which makes the approach even more complicated.

\section{Data Used in This Report}

In order to apply ray tracing techniques to test and understand what we discussed previously, atmospheric radiosonde data were used. Radiosonde data have reasonably vertical resolution within the troposphere and global coverage. For this report radiosonde data from about 640 stations were used, covering a period from January, 1990 to August, 1998. An approximate global distribution of the stations is given in Fig. 1, while the total number of radiosonde profiles is over 850000 .

\section{Preliminary Analysis and Results}

The results of preliminary analysis on a small portion of the data set show that the Chao model performs surprisingly well to low elevation angles for a lot of cases. This conclusion allows to introduce for the purpose of this work two models based on the analysis of the data used to develop the Ifadis global function (Ifadis, 1986, 1987) and keep the same fraction form

$$
m(\epsilon)=\frac{1}{\sin (\epsilon)+\frac{a}{\sin (\epsilon)+\frac{b}{\sin (\epsilon)+0.078}}}
$$

where $m(90)=1$ by definition. For the first of these models only temperature and water vapor pressure dependence is 
Table 3. Hydrostatic delay mismodeling due to pointing error for different mapping functions.

\begin{tabular}{ccccccccc}
\hline $\begin{array}{c}\text { Apparent } \\
\text { elevation } \\
\text { angle }\end{array}$ & $\begin{array}{c}\text { IF global } \\
(\mathrm{m})\end{array}$ & $\begin{array}{c}\text { IF climate } \\
(\mathrm{m})\end{array}$ & $\begin{array}{c}\text { MTT } \\
(\mathrm{m})\end{array}$ & $\begin{array}{c}\text { Marini } \\
(\mathrm{m})\end{array}$ & $\begin{array}{c}\text { CHAO } \\
(\mathrm{m})\end{array}$ & $\begin{array}{c}\text { NMF } \\
(\mathrm{m})\end{array}$ & $\begin{array}{c}\text { IF NEW } \\
(\mathrm{m})\end{array}$ & $\begin{array}{c}\text { IF CONST } \\
(\mathrm{m})\end{array}$ \\
\hline 10.000 & 0.001 & 0.001 & 0.000 & 0.000 & 0.000 & 0.000 & 0.000 & 0.000 \\
7.000 & 0.000 & 0.000 & 0.000 & 0.000 & 0.001 & 0.000 & 0.000 & 0.001 \\
6.000 & 0.000 & 0.001 & 0.001 & 0.001 & 0.001 & 0.001 & 0.001 & 0.001 \\
5.000 & 0.002 & 0.001 & 0.002 & 0.002 & 0.001 & 0.001 & 0.002 & 0.001 \\
4.000 & 0.002 & 0.003 & 0.003 & 0.003 & 0.003 & 0.003 & 0.003 & 0.003 \\
3.500 & 0.004 & 0.004 & 0.004 & 0.004 & 0.004 & 0.004 & 0.004 & 0.004 \\
3.000 & 0.006 & 0.006 & 0.006 & 0.005 & 0.006 & 0.006 & 0.005 & 0.006 \\
2.500 & 0.009 & 0.009 & 0.008 & 0.006 & 0.009 & 0.009 & 0.009 & 0.009 \\
2.000 & 0.013 & 0.013 & 0.014 & 0.008 & 0.014 & 0.014 & 0.013 & 0.014 \\
1.500 & 0.022 & 0.022 & 0.023 & 0.004 & 0.021 & 0.025 & 0.022 & 0.023 \\
1.000 & 0.039 & 0.039 & 0.043 & -0.017 & 0.030 & 0.050 & 0.040 & 0.040 \\
0.500 & 0.083 & 0.083 & 0.098 & -0.069 & 0.030 & -162.478 & 0.084 & 0.084 \\
\hline
\end{tabular}

Table 4. Hydrostatic delay residuals $(\mathrm{m})$ for a cold temperate station.

\begin{tabular}{|c|c|c|c|c|c|c|c|c|}
\hline $\begin{array}{c}\text { Apparent } \\
\text { elevation } \\
\text { angle }\end{array}$ & $\begin{array}{c}\text { IF-GLOBAL } \\
(\mathrm{m})\end{array}$ & $\begin{array}{l}\text { IF-climate } \\
(\mathrm{m})\end{array}$ & $\begin{array}{l}\text { IF-NEW } \\
(\mathrm{m})\end{array}$ & $\begin{array}{l}\text { MTT } \\
(\mathrm{m})\end{array}$ & $\begin{array}{l}\mathrm{NMF} \\
(\mathrm{m})\end{array}$ & $\begin{array}{c}\text { IF-CONST } \\
(\mathrm{m})\end{array}$ & $\begin{array}{l}\mathrm{CHAO} \\
(\mathrm{m})\end{array}$ & $\begin{array}{l}\text { MARINI } \\
(\mathrm{m})\end{array}$ \\
\hline 80 & 0.003 & 0.003 & 0.003 & 0.000 & 0.000 & 0.003 & 0.000 & 0.000 \\
\hline 70 & 0.003 & 0.003 & 0.003 & 0.000 & 0.000 & 0.003 & 0.001 & 0.001 \\
\hline 60 & 0.004 & 0.004 & 0.004 & 0.000 & 0.000 & 0.003 & 0.002 & 0.001 \\
\hline 50 & 0.004 & 0.004 & 0.004 & 0.000 & 0.000 & 0.004 & 0.002 & 0.001 \\
\hline 40 & 0.005 & 0.005 & 0.005 & 0.000 & 0.000 & 0.005 & 0.003 & 0.001 \\
\hline 30 & 0.006 & 0.006 & 0.006 & 0.000 & 0.000 & 0.005 & 0.004 & -0.001 \\
\hline 20 & 0.009 & 0.009 & 0.009 & 0.000 & -0.001 & 0.007 & 0.008 & -0.004 \\
\hline 18 & 0.010 & 0.010 & 0.010 & 0.000 & -0.001 & 0.007 & 0.008 & -0.007 \\
\hline 12 & 0.017 & 0.016 & 0.016 & 0.001 & -0.004 & 0.008 & 0.009 & -0.024 \\
\hline 10 & 0.021 & 0.020 & 0.020 & 0.001 & -0.006 & 0.007 & 0.004 & -0.040 \\
\hline 9 & 0.023 & 0.023 & 0.023 & 0.001 & -0.009 & 0.005 & -0.003 & -0.052 \\
\hline 8 & 0.027 & 0.025 & 0.026 & 0.001 & -0.012 & 0.001 & -0.015 & -0.068 \\
\hline 7 & 0.031 & 0.029 & 0.029 & 0.001 & -0.018 & -0.005 & -0.039 & -0.088 \\
\hline 6 & 0.036 & 0.034 & 0.034 & 0.000 & -0.026 & -0.015 & -0.084 & -0.106 \\
\hline 5 & 0.044 & 0.041 & 0.041 & 0.000 & -0.040 & -0.034 & -0.169 & -0.101 \\
\hline 4 & 0.058 & 0.053 & 0.053 & 0.001 & -0.066 & -0.067 & -0.336 & 0.030 \\
\hline 3 & 0.087 & 0.077 & 0.078 & -0.001 & -0.130 & -0.124 & -0.647 & 0.749 \\
\hline 2 & 0.128 & 0.112 & 0.112 & -0.071 & -0.358 & -0.222 & -1.022 & 3.741 \\
\hline 1.5 & 0.085 & 0.064 & 0.062 & -0.406 & -1.015 & -0.398 & -0.909 & 10.398 \\
\hline 1 & -0.349 & -0.375 & -0.382 & -1.657 & -3.093 & -0.949 & 0.659 & 24.028 \\
\hline
\end{tabular}

used (i.e. total pressure is not used) and

$$
\begin{aligned}
& a=K_{1 a}+K_{2 a}\left(T_{0}-15\right)+K_{3 a} \sqrt{ } p w \\
& b=K_{1 b}+K_{2 b}\left(T_{0}-15\right)+K_{3 b} \sqrt{ } p w
\end{aligned}
$$

This model is referred in the followings as IF-NEW. The second is similar to Chao (but with three terms) and constant values are used for the $a, b, c$ parameters. It is referred to as IF-CONSTANT.

\section{Results and Conclusions}

In this report some things concerning the atmospheric delay and the mapping functions were discussed and tested.
First, the error due to the finite distance of earth orbiting satellites was calculated, and, second, the sensitivity of the delay on standard geographical parameters was also tested. In order to get reliable results, ray tracing analysis was performed using data from all the stations shown in Fig. 1.

In ray trace we used the elevation angle of the radio source in two ways, first assuming the source is at infinity and second calculating the elevation of a radio source at GPS height and at low orbit height under the same conditions. These two slightly different elevations were applied for each of the mapping functions, and the differences in the predicted delays are given in Table 3 . It can be seen that for radio sources at GPS height or greater the difference is less than 1 


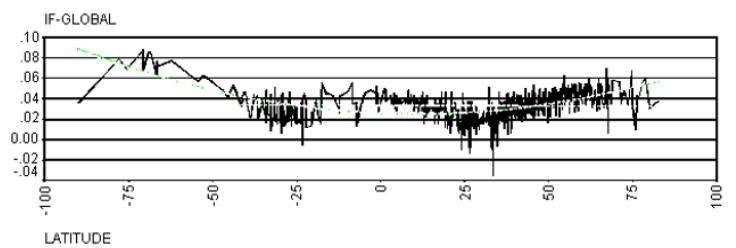

iF-CONSTANT
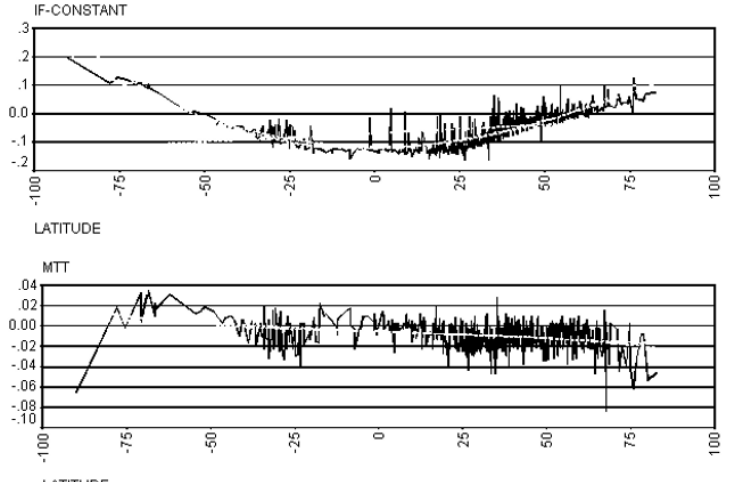

LATITUDE

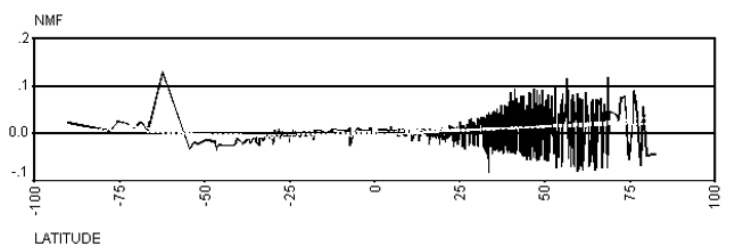

Fig. 2. Mapping functions delay residuals at $5^{\circ}$ elevation versus station latitude.

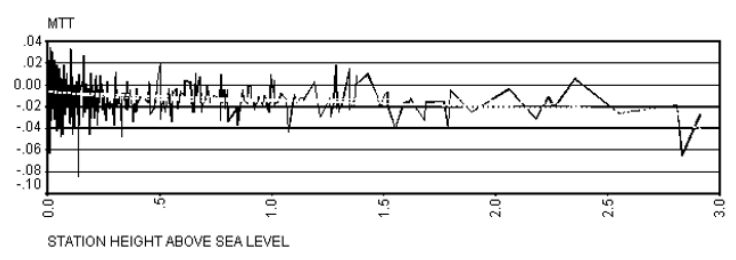

IF-CONSTANT

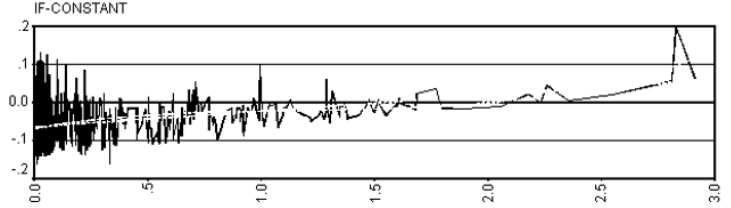

STATION HEIGHT ABOVE SEALEVEL

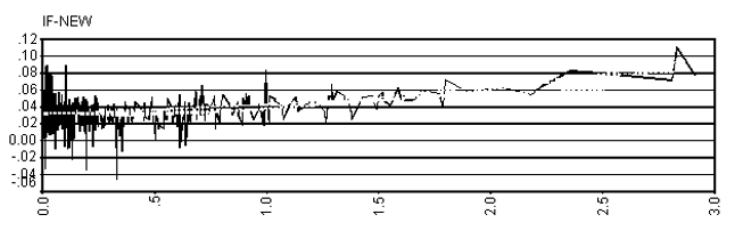

STATION HEIGHT ABOVE SEA LEVEL

Fig. 3. Mapping functions delay residuals at $5^{\circ}$ elevation versus station height.

$\mathrm{cm}$ at elevations greater than $3^{\circ}$. However for low earth orbits the error can be significant. In any case the results presented here are a first approximation and will not be analyzed any further.

Another purpose of this report was to test how geographical parameters can affect the mapping function accuracy. For
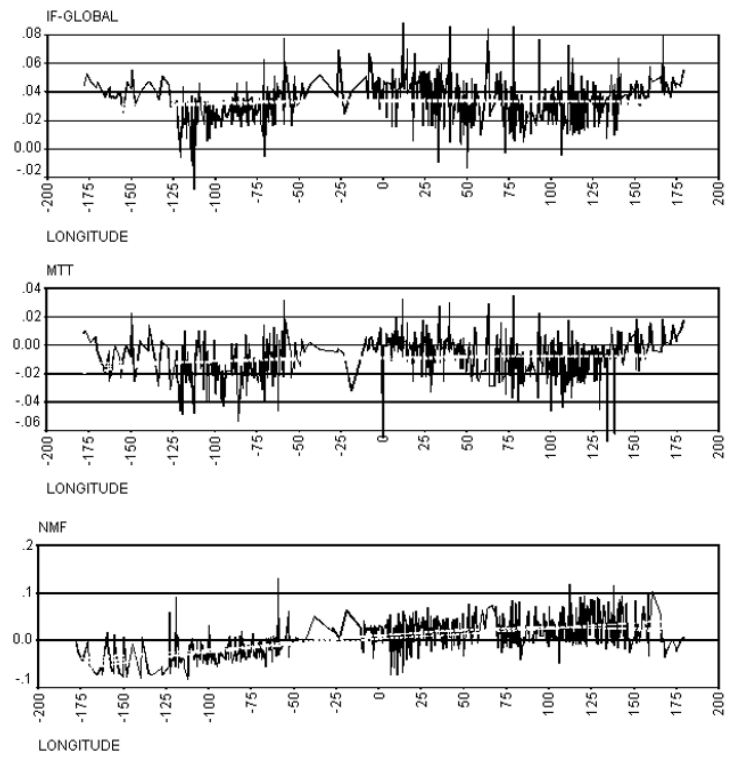

Fig. 4. Mapping functions delay residuals at $5^{\circ}$ elevation versus station longitude.

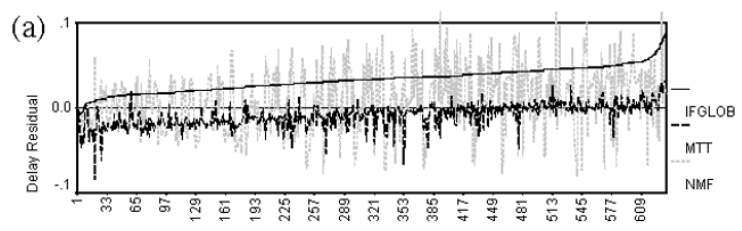

Case Number

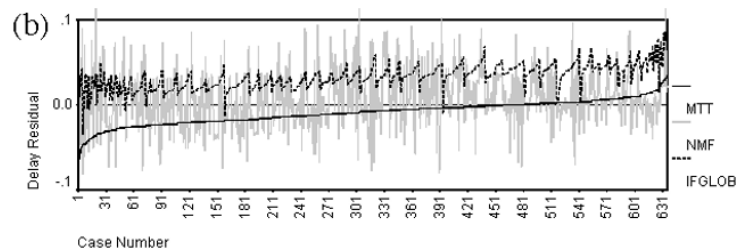

Case Number

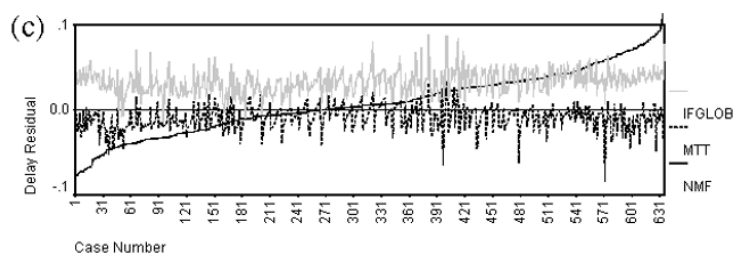

Fig. 5. Delay residuals $(\mathrm{m})$ at $5^{\circ}$ sorted from lower to higher values (solid black line) according to residuals given by a) Ifadis, b) MTT, and c) NMF mapping functions. a) The IF-GLOBAL model residuals sorted ascending (solid line). The dashed line represents the MTT model residuals and the gray dot-line the NMF model residuals. b) The MTT model residuals sorted ascending (solid line). The dot-line represents the IF-GLOBAL model residuals and the gray line the NMF model residuals. c) The NMF model residuals sorted ascending (solid line). The dot-line represents the MTT model residuals and the gray line the IF-GLOBAL model residuals.

this reason we plotted the delay residuals for $5^{\circ}$ and $2^{\circ}$ elevation angles against station latitude, longitude, and height. Some of these plots are given in Figs. 2, 3, and 4. There it is clear that the models with constant terms (even if the IF-CONSTANT has a better parameter optimization) cannot approximate well the delay at low elevation angles, at least 

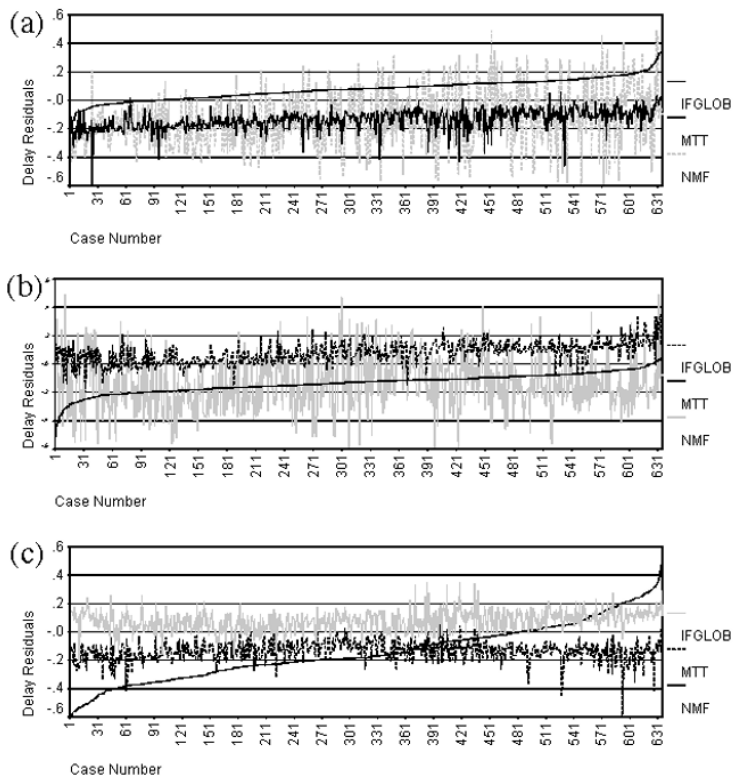

Fig. 6. Delay residuals $(\mathrm{m})$ at $2^{\circ}$ sorted from lower to higher values (solid black line) according to residuals given by a) Ifadis, b) MTT, and c) NMF mapping functions. a) The IF-GLOBAL model residuals sorted ascending (solid line). The dashed line represents the MTT model residuals and the gray dot-line the NMF model residuals. b) The MTT model residuals sorted ascending (solid line). The dot-line represents the IF-GLOBAL model residuals and the gray line the NMF model residuals. c) The NMF model residuals sorted ascending (solid line). The dot-line represents the MTT model residuals and the gray line the IF-GLOBAL model residuals.

in the way and form they were used in this report.

Some other useful conclusions can also be derived.

a) There is a large latitude dependence for the models with constant terms, while there seems to exist a slight dependence also for the IF-GLOBAL and IF-NEW models. The NMF model gives the lower bias averaged for all sites available (see also Figs. 5 and 6) but what is surprising is the high residual values for stations in the northern hemisphere. These results are inconsistent with the statistics found in the original paper where the NMF model was tested against Ifadis-global and MTT models using results from 26 sites from the northern hemisphere.

b) A large height dependence exists for the Chao and IFCONSTANT models as expected, but also for the IFNEW model. If we go back to the definition of this model we can see that the only difference from the IFGLOBAL is the absence of the pressure dependence. Thus it can be said that even a weak dependence on pressure should be included. The MTT model has been parameterized against station height above sea level but looking at Fig. 2 we can observe that there is a slight dependence of the residuals on station height. Then we can relate this with the fact that the surface total pressure does not always correlate well with the station altitude.

c) There is also a possible longitudinal dependence of the mapping function models as seen from Fig. 4 (especially for the NMF model which has no atmospheric dependence). Perhaps this is an unmodeled error, but it is not clear where it comes from.

In Figs. 5 and 6 there are plots of the delay residuals given by three mapping functions (Ifadis Global, MTT, and NMF). For each model the residuals were sorted ascending and then plotted together with the relative residuals given by the other two models. It can be seen that the IF-GLOBAL model overestimates the delay for most of the cases while the MTT model underestimates it and could be interesting to search why these two models give, in a way opposite results. The $\mathrm{NMF}$ model above $5^{\circ}$ gives low mean delay error values, but as the elevation angle decreases its accuracy also decreases (see Table 4). Also from Table 4 we can see that the IFCONST model, which has constant values for its parameters, predicts the delay with satisfactory accuracy, at least for the case of the station studied in this table. This kind of model has not been studied carefully yet, but it is believed that for certain regions, if optimized, will give accepted results even for low elevation angles.

Another careful conclusion that can be derived is that perhaps the continued fraction form of the MTT/NMF models gives a better geometrical approximation of the elevation dependence of the delay than the simple Chao fraction form. This assumption, together with better parameterization might give better results as indicated by reduced scatter plot of the delay residuals. Even if parameters such as station latitude and height can approximate and replace the mean atmospheric conditions in an area, then cannot predict and work for any special occasion as well as atmospheric parameters.

Acknowledgments. I sincerely wish to acknowledge my family for the support and patience, my colleagues at the Section of Geodesy for taking the effort of teaching hours during my leave, and finally the British Atmospheric Data Center for allowing the download of the large amount of data used in this research project.

\section{References}

Chao, C. C., A new method to predict wet zenith range refraction from surface measurements of meteorological parameters, in DSN Progress Report, No. 32-1526, Vol. XIV, pp. 33-41, Jet Prop. Lab., Pasadena, California, 15 April 1973, 1973.

Davis, J. L., T. A. Herring, I. I. Shapiro, A. E. E. Rogers, and G. Elgered, Geodesy by radio interferometry: Effects of atmospheric modeling errors on estimates of baseline length, Radio Sci., 20, 1985.

Herring, T. A., Modeling atmospheric delays in the analysis of space geodetic data, in Proceedings of the Symposium on Refraction of Transatmospheric Signals in Geodesy, edited by J. C. de Munck and T. A. Th. Spoelstra, pp. 157-164, The Netherlands, 19-22 May, Netherlands Geodetic Commission, Publications on Geodesy, Delft, The Netherlands, No. 36, New Series, 1992

Ifadis, I. The Atmospheric Delay of Radio Waves: Modelling The Elevation Dependence on a Global Scale, Licentiate Thesis, Technical Report No 38L, Chalmers University of Technology, Gothenburg, Sweden, 1986.

Ifadis, I., Contribution to the Study of the Atmospheric Refraction, on Radio Waves Used in Modern Geodetic Techni-ques in Long Distance Measurements, Thesis Report, vol. IA'.6, School of Engineering, Faculty of Civil Engineering, Thessaloniki, 1987.

Ifadis, I. and P. Savvaidis, Review of Mapping Functions Proposed to Predict the Delay at Very Low Elevation Angles, Proceedings of the 55th ION meeting, Cambridge, Ma, June 28-30, 1999.

Marini, J. W., Correction of satellite tracking data for an arbitrary atmospheric profile, Radio Sci., 7, 223-231, 1972.

Niell, A. E., Global mapping functions for the atmospheric delay at radio wavelengths, J. Geophys. Res., 101(B2), 3227-3246, 1996.

I. M. Ifadis (e-mail: ifadis@civil.auth.gr) 